\title{
La construcción de una bóveda de crucería en la Escuela Técnica Superior de Arquitectura de Madrid
}

\author{
Construction of a ribbed vault at the Escuela Técnica \\ Superior de Arquitectura in Madrid
}

\author{
L.C. Palacios ${ }^{(*)}$, R. Martín(*)
}

\section{RESUMEN}

En la Escuela Superior de Arquitectura de Madrid, el Departamento de Construcción ha puesto en marcha una experiencia pedagógica de extraordinario interés. En el marco de una nueva asignatura Ilamada Taller de Construcción Gótica, los alumnos han podido llevar a cabo la construcción de una bóveda de crucería.

Las bóvedas "góticas" son objeto de estudio desde distintos puntos de vista, sin embargo, los conocimientos adquiridos en estas áreas jamás se confrontan con la práctica real. Esta experiencia pedagógica propone invertir los términos, es decir, redescubrir y dar un sentido a la teoría a través de la experiencia física de la construcción.

Durante su construcción se fueron poniendo de relieve todos los problemas inherentes a este tipo de construcciones; en primer lugar el conocimiento histórico del objeto y su autor, su concepción geométrica y el descubrimiento de su forma esférica. A continuación dio comienzo la talla de las diferentes piezas que componen la bóveda, y seguidamente la construcción de las cimbras que, una vez acabadas permitieron llevar a cabo el montaje de la bóveda. Finalmente se llevó a cabo el descimbrado de la misma.

Sobre todo este proceso, trata el artículo que a continuación se presenta. Cualquier conocimiento teórico queda rebasado por la riqueza de la experiencia de su puesta en práctica real.

\section{3-11}

Palabras clave: bóveda, arco, nervio, jarja, clave.

\section{SUMMARY}

The Department of Construction in the Escuela Superior de Arquitectura de Madrid carried out a pedagogical experience of great interest. In the framework of a new discipline (Workshop on Gothic Art of Construction), the students built a ribbed vault.

Gothic vaults are studied from several points of view, but the knowledge acquired in all these fields is never confronted with real practice. The aim of this pedagogical experience was to invert the terms, that is to rediscover and instil meaning into the theory through the physical experience of the construction.

Along the process, the students were made conscious of the following aspects inherent to this type of construction: first of all, we planed to acquire historical knowledge of the object and its author; second, we took up its geometric study and the discovery of its spherical shape, and, third, we carried out the design of its life-size geometric lay out. In the next stage, we proceeded to carve the different parts of the vault, to pass on to build the centering which, once finished, enabled us to tackle the assembly of the vault. Finally, we removed the timber framework of the vault.

This article deals with this whole process. The students who carried out the experiment of building a real vault can attest how to upgrade theoretical knowledge through actual experience is the best way to learn.

Keywords: vault, arch, rib, springer's fork, keystone. 
El pasado curso, en la Escuela Superior de Arquitectura de Madrid, el Departamento de Construcción puso en marcha una experiencia pedagógica de extraordinario interés. En el marco de una nueva asignatura Ilamada Taller de Construcción Gótica, los alumnos han podido llevar a cabo la construcción de una bóveda de crucería de diecisiete claves. La bóveda construida es la que aparece en el manuscrito del s. XVI atribuido a Alonso de Vandelvira "Libro de las traças e cortes de cantería".

Los objetivos que con esta iniciativa se pretendían lograr eran diversos, todos ellos, habrían de alcanzarse vinculando la teoría a la práctica con la construcción real de una bóveda de crucería. En la Escuela de Arquitectura de Madrid, las bóvedas "góticas" son objeto de estudio desde distintos puntos de vista: la historia, la construcción, las estructuras, la geometría etc., sin embargo, los conocimientos adquiridos en estas áreas jamás se confrontan con la práctica real. Esta experiencia pedagógica propone invertir los términos, es decir, redescubrir y dar un sentido a la teoría través de la experiencia física de la construcción; veamos cómo se produce entonces esta amalgama de conocimientos.

En primer lugar, la construcción de la bóveda nos introduce en un periodo histórico concreto, el complejo mundo del gótico tardío español se extiende ante nosotros de la mano de una figura de primera magnitud de nuestro renacimiento: Vandelvira. A través de él, seremos espectadores de ese periodo irrepetible de la arquitectura española, en el que la arquitectura clásica y el último gótico alcanzan el momento de máximo desarrollo en nuestro país. Por otra parte, la construcción de la bóveda exige que sus trazas geométricas sean exploradas en profundidad, éste proceso llevará a descubrir las herramientas geométricas de los maestros de cantería del XVI. El abordaje de la geometría de la bóveda se va a llevar a cabo desde dos puntos de vista, el contemporáneo, mediante el uso del ordenador, y el histórico, ejecutando sus monteas a tamaño natural como era práctica habitual desde la Edad Media. La construcción de la bóveda nos interroga también sobre la estabilidad de las estructuras históricas, su puesta en obra, permite sentir físicamente cómo las cargas se desplazan a través de los arcos creando empujes horizontales y posibles tracciones en las secciones de los arcos. Comprender las acciones que las cargas generan sobre la bóveda lleva a examinar los mecanismos con los tradicionalmente se contrarrestaban: contrafuertes, estribos, rellenos, etc.

Por último, la construcción de la bóveda nos sumerge en el mundo de la cantería medieval en un momento, en el siglo XVI, en que la estereotomía renacentista acaba de aparecer. Al Ilevar a cabo la talla de dovelas y claves se van desvelando las técnicas medievales de corte y labra mediante las cuales la abstracta geometría de los arcos y la volumetría de las piezas más complejas se trasladaban a la piedra. Esta tarea nos introduce además en el mundo de las estructuras gremiales de la Edad Media y, en cierto modo, a participar de la unión y solidaridad profesional que se genera con el trabajo en equipo, consustancial a los talleres de cantería medievales. Ni que decir tiene que la experiencia de construir una bóveda gótica compleja esta hoy día alejada por completo del horizonte pedagógico que puede encontrar un alumno de arquitectura; sin embargo, por las razones que acabamos de exponer, podría ser un camino interesante para que los conocimientos teóricos parciales que sobre este tema el alumno va adquiriendo a lo largo de su carrera, terminen afirmándose con la experiencia concreta. Una experiencia que, colateralmente, abre al alumno las puertas del apasionante mundo de la restauración y conservación de monumentos.

\section{EL MANUSCRITO Y LA BÓVEDA DE VANDELVIRA}

Como mencionamos anteriormente, la bóveda elegida para su construcción no es una bóveda real, jamás fue construida, se trata de un modelo teórico que aparece en el libro manuscrito de Alonso de Vandelvira, sin duda alguna, uno de los libros de arquitectura más singulares de la bibliografía española de arquitectura. Una copia de este libro se encuentra actualmente en la biblioteca de la Escuela de Arquitectura de Madrid (1).

Los Vandelvira fueron una familia de arquitectos de la provincia de Jaén, el padre de Alonso fue el célebre Andrés de Vandelvira, uno de los gigantes del gótico español, arquitecto de la catedral de Jaén y de una importante cantidad de edificios en úbeda y Baeza con los que el renacimiento español adquirió su peculiar e irrepetible fisonomía.

El mencionado manuscrito esta dedicado a la cantería y al arte de construir en piedra todo tipo de arcos y bóvedas. Los elementos arquitectónicos que se estudian en este libro corresponden a una arquitectura clásica, es decir, una arquitectura "a lo romano", la única bóveda de crucería que contiene es la que hemos seleccionado para llevar a cabo su construcción. Se trata de una bóveda singular, por así decir, un prototipo (Figura 1), con él Vandelvira quiere mostrar cómo construir una bóveda gótica de forma esférica. Su forma redonda permite una novedosa traza de combados en forma de ruedas concéntricas, este 
dibujo sería de imposible ejecución en los quebrados témpanos de plementería de una bóveda gótica tradicional. La bóveda se forma con 17 claves y tres tipos de arcos: ojivo, tercelete y fajón. Las jarjas de la bóveda son también interesantes ya que, en lugar de que el arranque se forme con un protuberante haz de nervaduras, por entonces la solución más frecuente, Vandelvira propone que los arcos se fundan entre sí hasta desaparecer fuera de los límites de la bóveda (2).

El conocimiento previo del prototipo de Vandelvira requirió un estudio geométrico detallado de su forma, con tal finalidad se llevaron a cabo diversos dibujos en tres dimensiones que en seguida revelaron la belleza de la bóveda (Figura 2). Posteriormente se trazaron las curvaturas de sus arcos y el completo despiece de las partes fundamentales de la bóveda: dovelas, claves y jarjas (Figura 3).

Como mencionamos anteriormente, al tratarse de una superficie esférica, los tres arcos con que se construye la bóveda, el ojivo, el tercelete y el fajón, se forman por la sección de esta superficie con tres planos distintos por lo que, los tres arcos, tienen curvaturas diferentes. Como veremos posteriormente, esta circunstancia afectará directamente a la talla de dovelas y a la construcción de los camones de las cimbras.

A partir de los dibujos tridimensionales es posible extraer la forma exacta de cada una de las claves y comprender la complejidad de cada una de estas piezas; su imagen tridimensional CAD permitiría además su

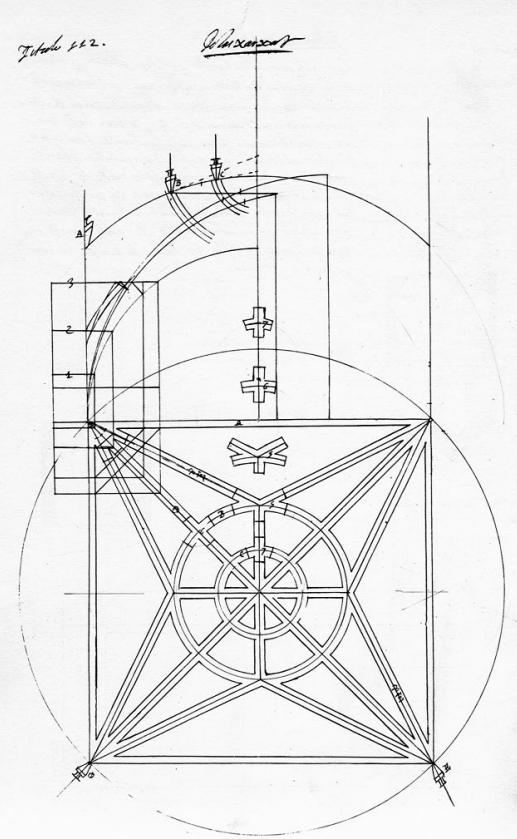

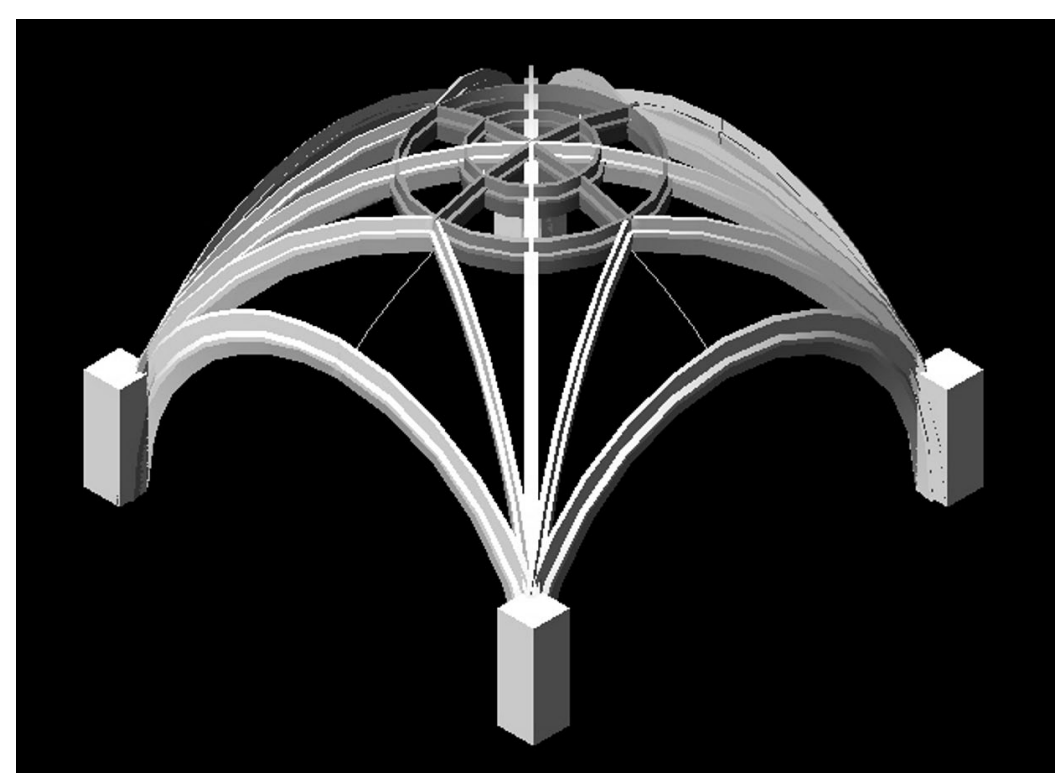

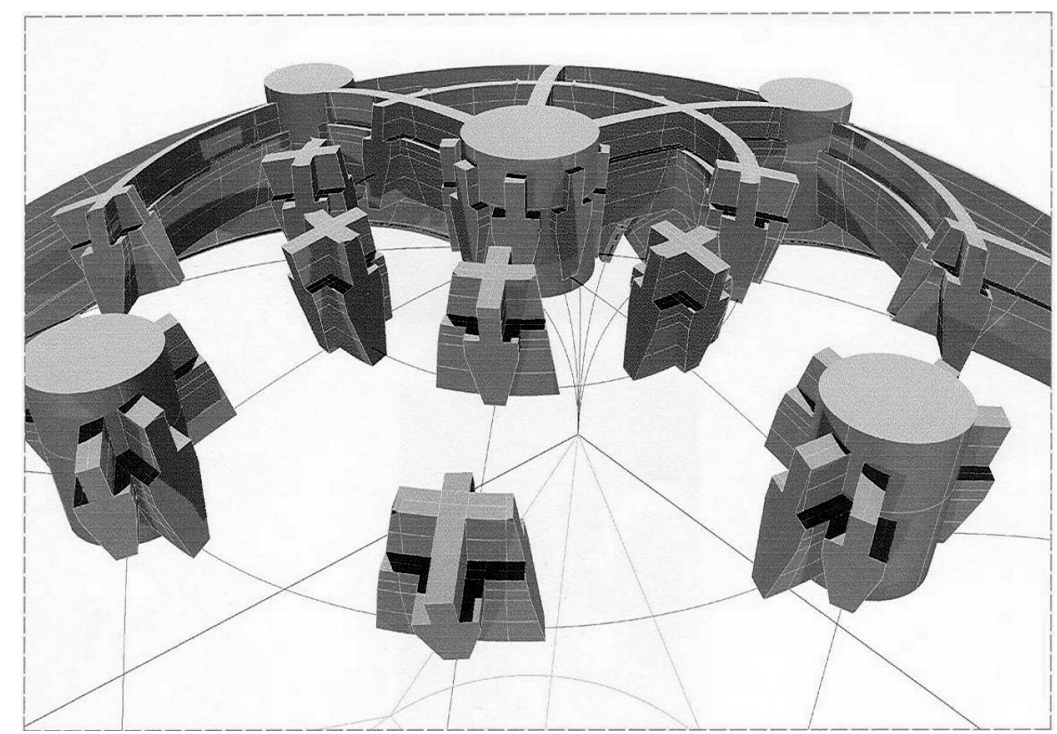

talla directa en piedra mediante máquinas de corte por control numérico.

Por último, se efectúa un estudio detallado de los arranques de la nervadura: las jarjas. Como es sabido, las bóvedas de crucería comienzan construyéndose por lechos horizontales hasta una altura variable, esta altura viene determinada por la cota en la que los arcos que concurren en un arranque se hacen independientes unos de otros.

En la mayor parte de los casos, las jarjas pueden alcanzar una altura cercana la mitad de la alzada de la bóveda. Los jarjamentos son elemento de la mayor importancia en la estabilidad de la bóveda ya que, al prolongarse los lechos de piedra horizontalmente, contribuyen en gran medida a encastrar la bóvedas en los muros o pilastras perimetrales (Figura 4).
1. Pagina del manuscrito de Alonso de Vandelvira en que aparece la bóveda de crucería seleccionada para ser construida.

2. Infografía tridimensional de la bóveda de Vandelvira en la que se aprecia claramente su forma esférica.

3. Imagen tridimensional del despiece de claves y cruceros (autor: Carmen Pérez de los Ríos) 


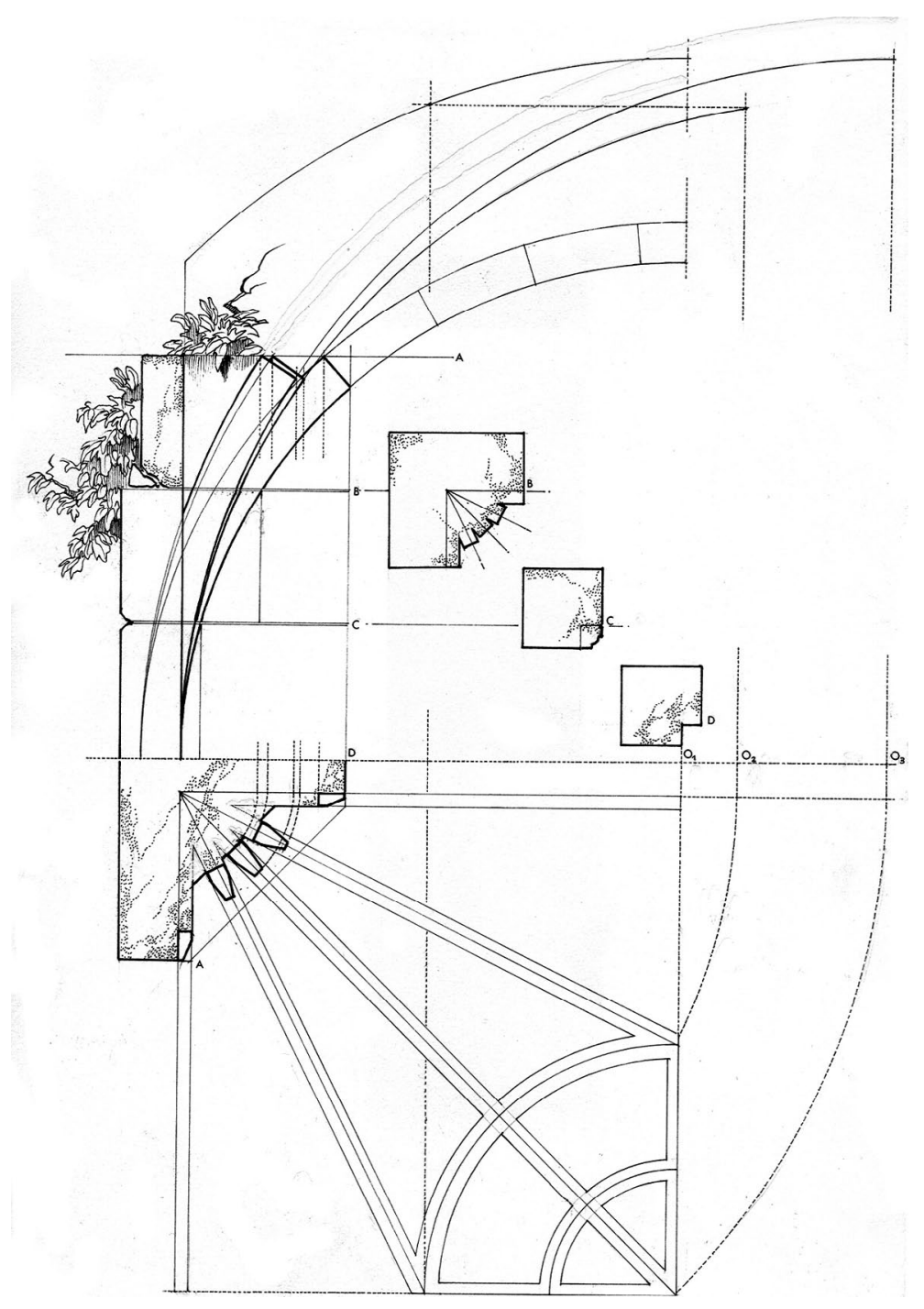

4. Las tres jarjas con sus plantillas de la bóveda de Vandelvira.

5. Dibujo de las monteas de la bóveda a tamaño natural.

6. De las monteas se extraen todas las medidas para la construcción de la bóveda.

1 Las placas de escayola han sido suministradas gratuitamente por la empresa YGARESA, de Torrelaguna, Madrid, empresa que viene colaborando desinteresadamente con este taller.
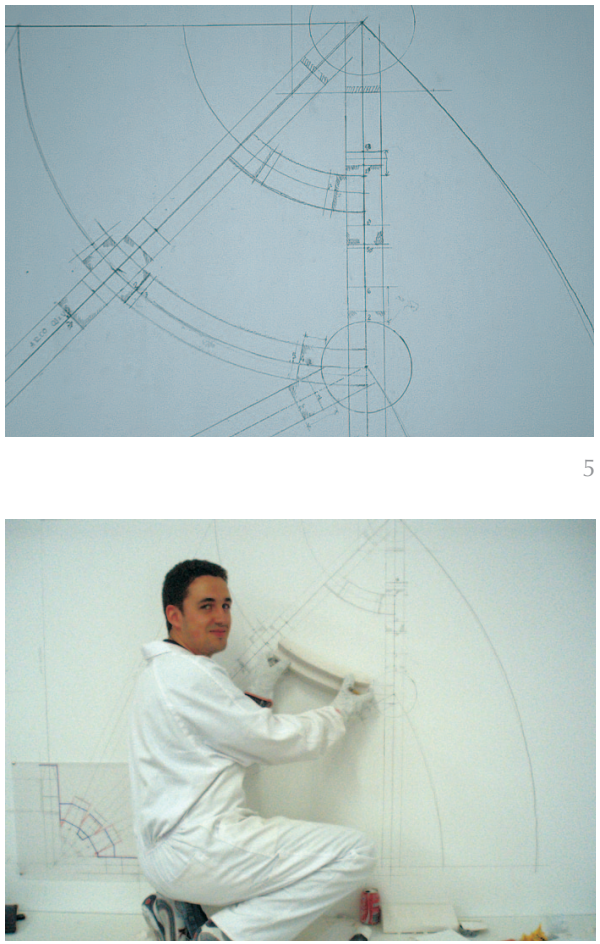

dibujo en planta de un objeto con su alzado o sección, fue desarrollándose en las logias y talleres de cantería nacidos a la sombra de las grandes catedrales desde la alta edad media. En el siglo XVI, la metodología permite ya resolver complicados abatimientos de arcos y el dibujo detallado de las piezas más complejas; ahora, como entonces, los datos necesarios para construir las diversas piezas que componen la bóveda se van a extraer de las monteas a tamaño natural (Figura 6).

Para la construcción de la bóveda se ha elegido como material la escayola en bloque, usándola como si fuera piedra, es decir cortándola y labrándola de la misma manera. Este material, usado también en la antigüedad para construir maquetas de estereotomía, permite una facilidad y rapidez de ejecución imposible de lograr con la piedra. Con la escayola se pretendía lograr que en el periodo de un curso -tres horas por semana durante cuatro meses- pudiera alcanzarse el objetivo de construir la totalidad de la bóveda contando con un total de cuarenta alumnos. Se usaron placas de escayola maciza de 6 $\mathrm{cm}$ de espesor de las que habitualmente se emplean para la construcción de tabiques' Respetando la escala del dibujo de Vandelvira, la medida del espesor de la placa de escayola nos determinaba las dimensiones de la bóveda, un cuadrado de de 2,40 m de lado y una altura de 1,80 m.

La construcción comienza con la labra de dovelas. Para empezar, a partir de la montea, se dibujan y confeccionan los baibeles, herramienta ésta imprescindible para controlar 


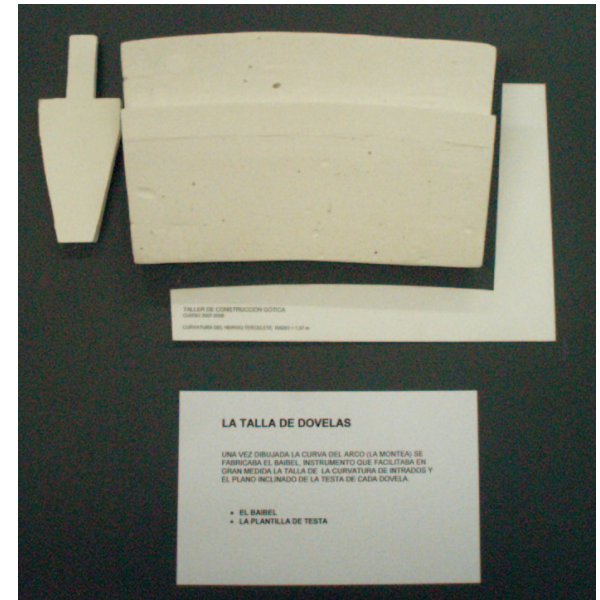

la talla. Se trataba de unas escuadras de dos brazos no articulados, uno de ellos cortado con la curvatura del intradós del arco y el otro, recto, orientado hacia el centro geométrico del arco (Figura 7). Como la bóveda tiene tres arcos diferentes, hemos de construir tres baibeles: para el ojivo, el tercelete y el fajón (Figura 8). En la bóveda de Vandelvira, la sección de los tres arcos es diferente, su autor explica escrupulosamente cómo revirar las secciones de los mismos para que se adapten mejor a la sección de la bóveda; sin embargo, en este caso, por simplificar, se ha decidido que la sección de los tres arcos sea idéntica, es decir, que los tres arcos tendrán una única plantilla para su sección. En la confección de esta plantilla de testa, se ha respetado la forma y, sobre todo, la escala que esta sección tiene en el dibujo de Vandelvira; también se ha respetado la cola, es decir, la protuberancia que lleva el trasdós del arco para que éste quede encastrado en la plementería. Finalmente, con el correspondiente baibel y la plantilla de testa, se pueden comenzar a tallar las dovelas, la escayola nos permitirá el uso del serrucho, el formón y la lima para alcanzar con bastante rapidez la forma deseada. A medida que las dovelas comienzan a aparecer se van almacenando ordenadamente.

Mencionamos anteriormente cómo la forma esférica de la bóveda permite a Vandelvira adornarla con un dibujo de combados consistente en dos ruedas concéntricas. Como es sabido, este tipo de nervadura decorativa subsidiaria recibe el nombre de combados.

Al observar detenidamente el dibujo de la sección de Vandelvira, se aprecia claramente cómo las testas de estos combados se deforman adaptándose a la posición que ocupan (Figura 9), de forma que, cuanto más alejados del centro, más deformada tienen su sección. Estas secciones torcidas, de "molde revirado" como se decía entonces, permitían recibir mejor los rellenos de la
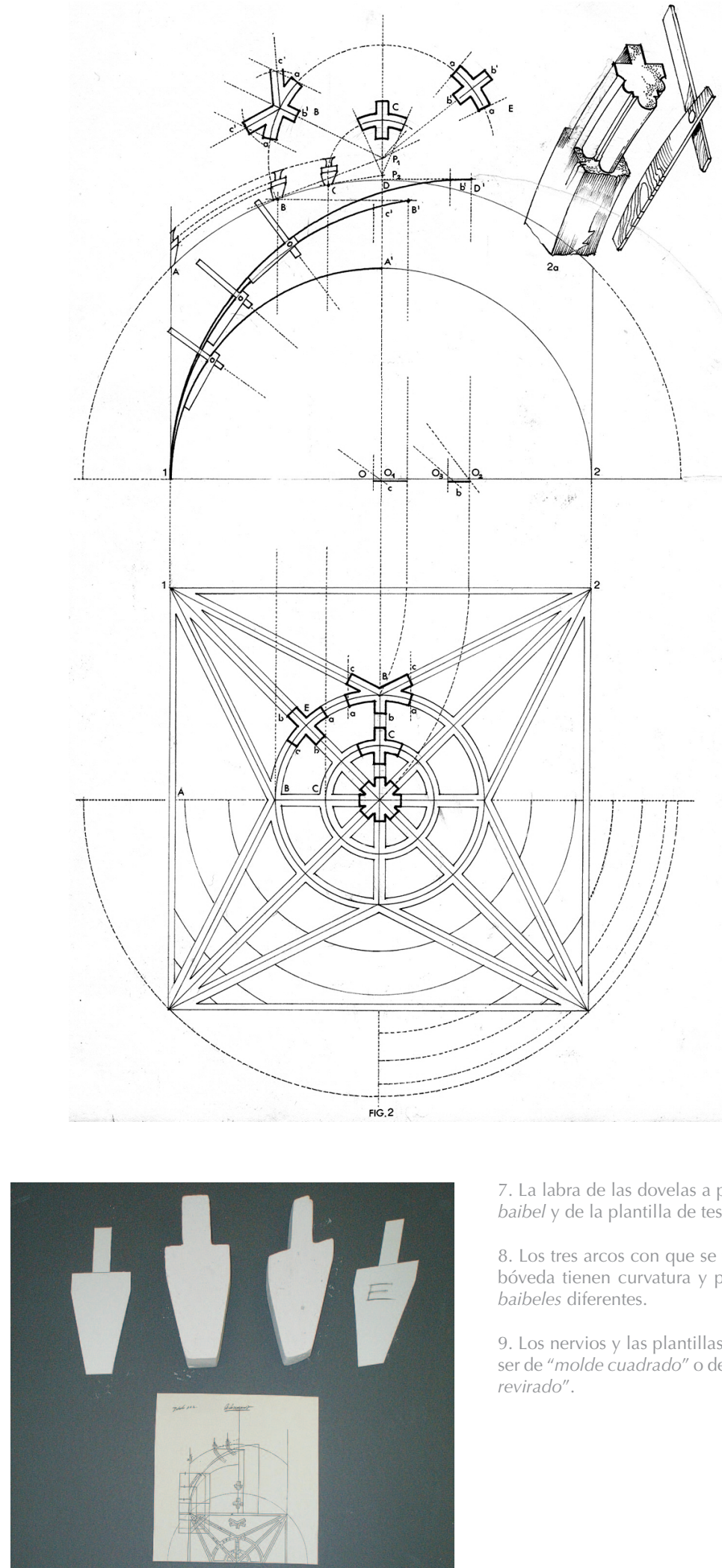

7. La labra de las dovelas a partir del baibel y de la plantilla de testa.

8. Los tres arcos con que se forma la bóveda tienen curvatura y por tanto baibeles diferentes.

9. Los nervios y las plantillas pueden ser de "molde cuadrado" o de "molde revirado". 
10. En primer lugar la talla de las claves se lleva a cabo por extrusión, a partir del dibujo de su proyección horizontal.

11. La parte más compleja de la talla de claves es el corte de las testas de los arcos que en ella confluyen

12. El corte de los ángulos de acometida es la parte más delicada en la talla de claves, estos ángulos se obtienen directamente de la montea.

13. La talla de los cruceros se efectúa a partir de los datos que se extraen de las monteas.

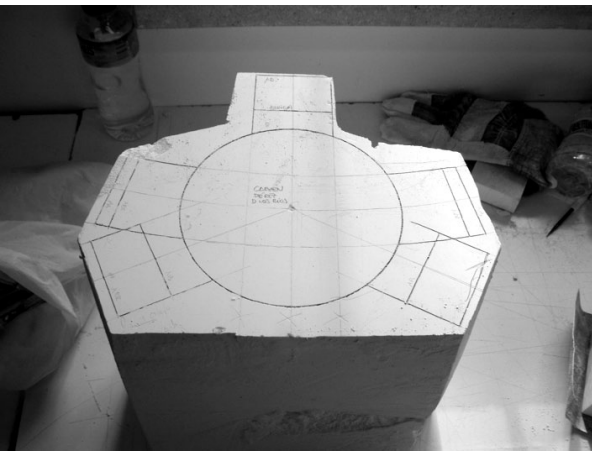

10

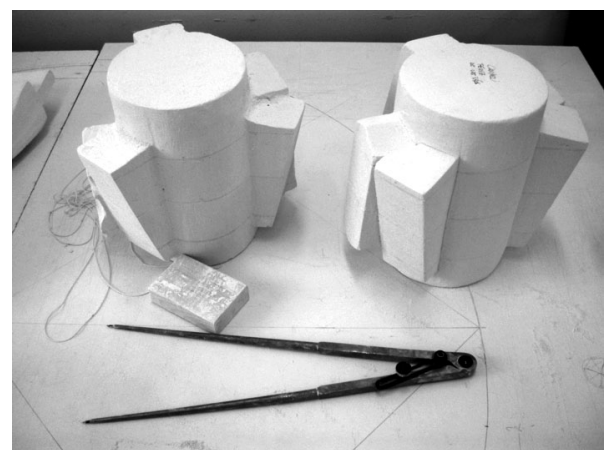

11
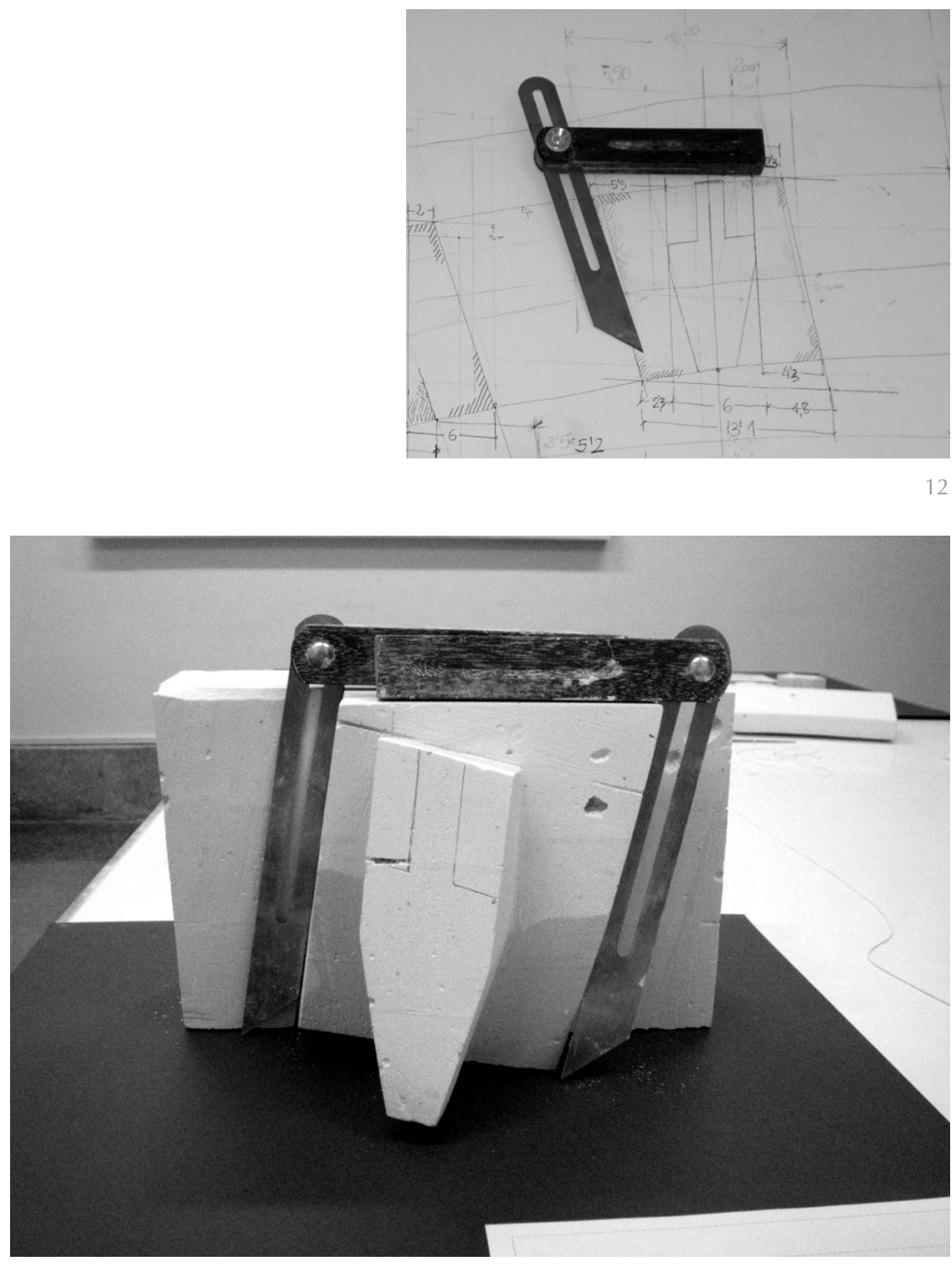

las que componen la bóveda, la talla de estos elementos ponía a prueba como ningún otro la capacidad de los maestros de cantería. Cuando se producía un cruce complicado entre varios arcos era frecuente interponer una pieza cilíndrica vertical, la clave, con esta pieza se simplificaba en gran medida el difícil encuentro entre los arcos. Sin embargo, cuando el encuentro es más sencillo, los arcos se intersectan limpiamente entre sí formando un crucero. En el dibujo de Vandelvira (Figura 1), la bóveda carece de claves, es decir, que todos los encuentros entre arcos se realiza por cruceros. En nuestro caso, para simplificar la construcción, se ha colocado una clave en el centro de la bóveda y en el encuentro de los terceletes.

Para llevar a cabo la labra de esta pieza hemos de volver de nuevo a la montea, la proyección horizontal de todas las claves y cruceros ha de dibujarse con precisión en el dibujo en planta de la bóveda (véase Figura 5) y, a partir de aquí, sus proyecciones en la sección. A continuación, sobre la cara superior de un bloque de piedra, se copia la proyección horizontal de la clave, posteriormente, mediante una labra por extrusión, se obtiene el sólido capaz de la clave o del crucero (Figura 10). Viene después la labor más delicada, se trata de cortar con la debida inclinación las testas de los arcos que concurren en esa clave para que ésta encaje correctamente (Figura 11). Para llevar a cabo este corte, hemos de conocer los ángulos de acometida de los arcos, estos ángulos deben obtenerse de la montea vertical de la bóveda es decir, del dibujo de los arcos abatidos y las claves que se sitúan sobre ellos. Como muestra la Figura 12, los ángulos de acometida se determinan en relación a un plano horizontal, ya sea el plano superior de la clave, o el inferior. Este ángulo se toma directamente de la montea mediante un compás de ángulos, para los antiguos una saltarregla. A continuación, este ángulo se trasladaba a la piedra y permitía dar el corte adecuado a las testas de cada arco. Por último, con las plantillas de testa, se daba forma a cada arco con lo que la labra de la clave había terminado (Figura 13). Obsérvese que, por regla general, los brazos de las claves son lo más cortos posible lo cual permite que no sea necesario tallar sus curvaturas (3).

\section{LOS JARJAMENTOS}

Para terminar, la bóveda requiere la construcción de unas sólidas jarjas. Como mencionamos anteriormente, las jarjas o salmeres de la bóveda son los arranques de la misma, estas piezas siempre se llevan a cabo mediante lechos horizontales. Sabemos que, en una bóveda de crucería las jarjas deben Ilegar hasta el punto en que los arcos que concurren en un arranque se independizan unos 
de otros. Esta altura puede determinarse mediante las proyecciones verticales y horizontales; cualquier plano horizontal trazado sobre la sección tiene inmediatamente una proyección en planta que permite ver si los arcos se han desgajados unos de otros, o si aún permanecen solidarios Los arranques de la bóveda de Vandelvira tienen una particularidad estética interesante, ya que no forman un haz prominente, como solía ser la solución más frecuente, sino que el conjunto de arcos desaparecen en los vértices de la bóveda. Esto significa que el arranque se produce más atrás, por así decirlo, fuera de los confines de la bóveda, como consecuencia, los nervios se van a independizar unos de otros más tarde; el resultado es que las jarjas de esta bóveda van a ser más altas de lo normal.

Para la construcción de las jarjas recurrimos de nuevo a la montea. Sobre la sección se van dibujando los planos horizontales que se consideren oportunos y, en la planta, se van dibujando las proyecciones horizontales resultantes en cada nivel (Figura 4). Con estas secciones se dibujan una serie de plantillas que permitirán tallar las piedras que forman cada lecho; la plantilla inferior se calca en el plano inferior del sillar elegido $y$, con la plantilla superior se hace lo mismo sobre la cara superior, posteriormente se procede a la labra enlazando una cara con la otra. Como la última jarja recibe los arcos, ha de tallarse con la inclinación necesaria que pide cada uno de ellos (Figura 14), dato éste que de nuevo ha de extraerse de la montea A medida que se van tallando cada uno de los lechos pueden irse colocando en su posición correcta, al colocar el lecho superior se aprecia claramente cómo las jarjas superan la mitad de la altura de la bóveda; su peso y el empotramiento que originan van a contribuir en gran medida a su estabilidad. El jarjamento de una bóveda suele pasar desapercibido, sin embargo, su construcción ha permitido apreciar claramente su importante volumen y el papel fundamental que juega en la construcción de la bóveda (Figura 15). Obsérvese que los arcos comienzan a funcionar como tales solamente cuando acaban las jarjas.

\section{LAS CIMBRAS}

Una vez que la labra va tocando a su fin llega el momento de la puesta en obra de la bóveda. Es entonces cuando, imperativamente, será necesario el concurso de la carpintería de armar para la confección de cimbras. El diseño de las cimbras requiere una cierta atención ya que deben soportar el peso de los arcos de piedra, a la vez, han de permitir un descimbrado correcto. En una bóveda de crucería, las cimbras pueden ser

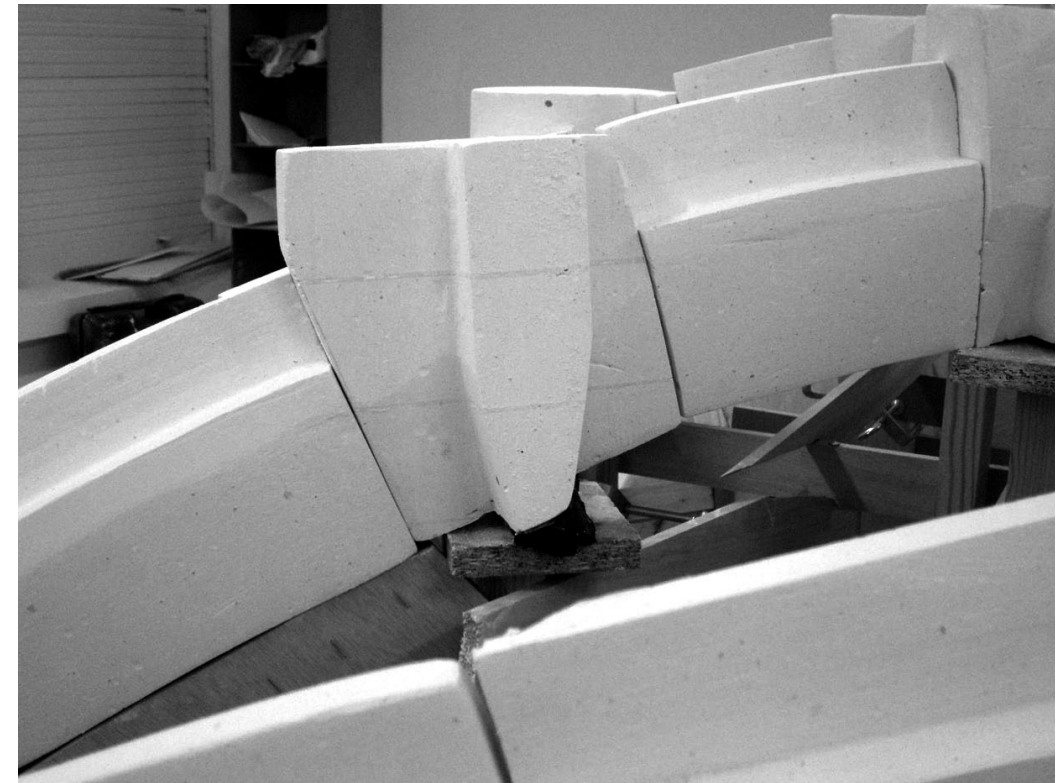

14

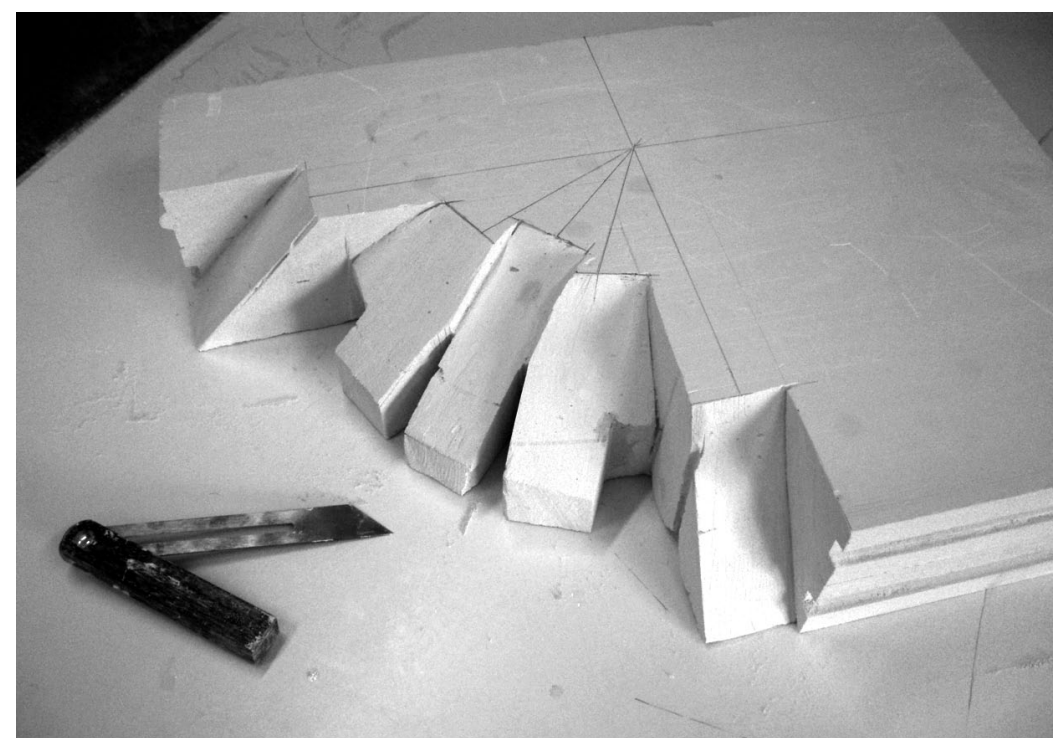

más ligeras que en una bóveda clásica de cáscara continua, ya que han de soportar únicamente el peso de los arcos de piedra; pensemos que, una vez que toda la crucería está montada, ésta puede hacerse cargo del peso de la plementería, por lo que la cimbra de una bóveda gótica no tiene que soportar el peso de la bóveda, una ventaja más del ingenioso sistema de abovedar gótico.

Para la construcción de la cimbra se han seguido los consejos que, en el siglo XVI, preconizaba a tal efecto Rodrigo Gil de Hontañón (Figura 16). Recomendaba en primer lugar la construcción de una plataforma de madera a la altura de las jarjas, sobre ella habría de dibujarse de nuevo la montea horizontal de la bóveda (4). En los puntos en que se situaban las claves se colocarían pies derechos con la altura adecuada para situar 


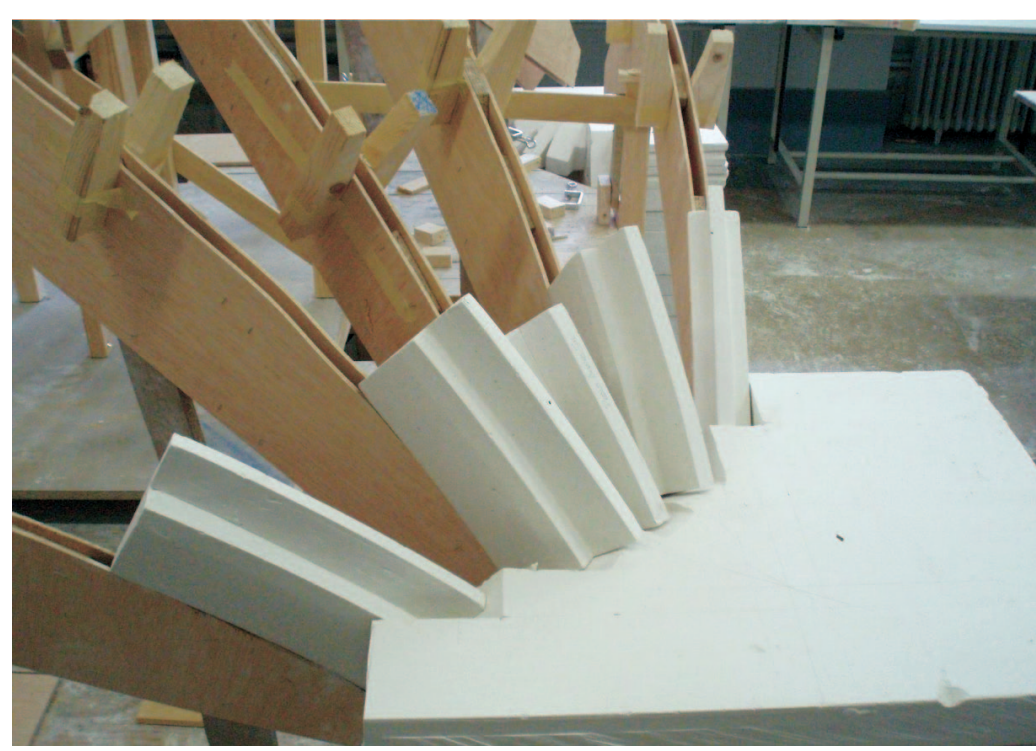

16

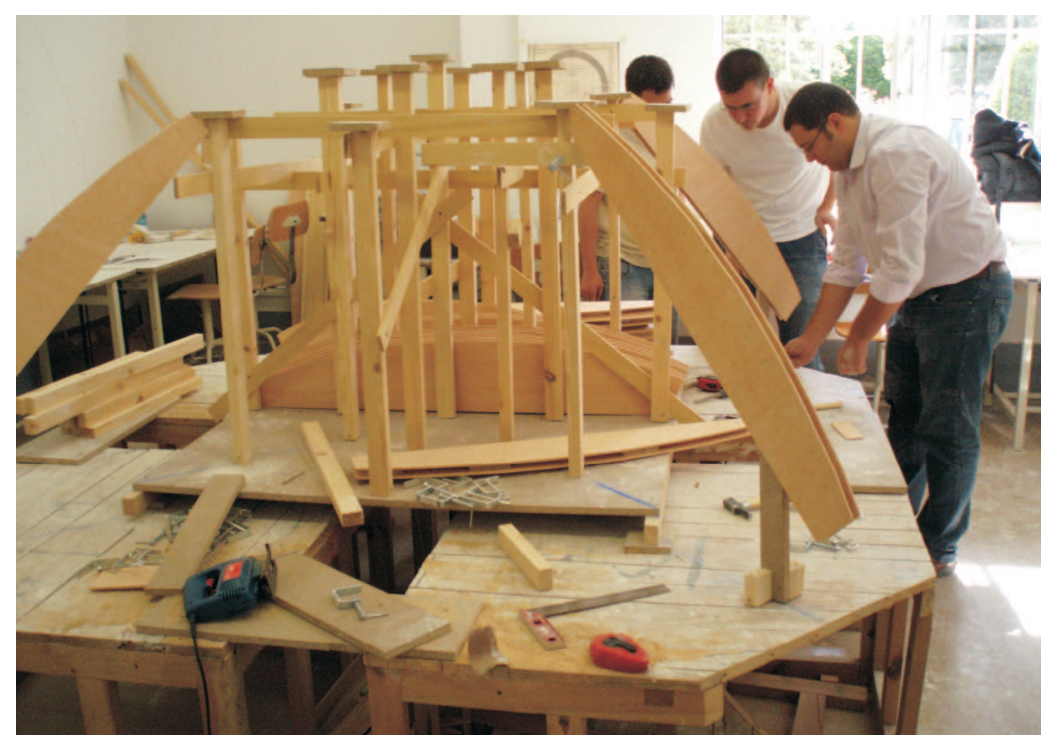

16. La bóveda propiamente dicha comienza cuando acaba el jarjamento.

17. La cimbra se compone de una gran plataforma que recibe los pies derechos situados bajo las claves $y$ los camones curvos de los arcos. en su posición cada una de ellas, esta altura se extrae, una vez más, de la montea a tamaño natural de la sección de la bóveda. Entre estos pies derechos se colocan las cimbras de los arcos, atando unos con otros y estabilizando el conjunto.

Habida cuenta que en la bóveda de Vandelvira, las 17 claves quedan arracimadas en el centro de la misma, los pies derechos se han situado a su vez sobre una plataforma más pequeña alzada mediante un sistema de cuñas; al extraer las cuñas, todas las claves descenderán a la vez de manera que, toda la bóveda, entrará en carga al mismo tiempo. Una vez construida la cimbra podemos apreciar en toda su dimensión la enorme envergadura de estas obras de carpintería, valorando el elevadísimo coste de las mismas y la importancia de reducirla al máximo. Con las cimbras acabadas se pueden ir colocando sobre ellas las dovelas y claves (Figura 17), es entonces cuando un nuevo problema requiere la máxima atención: las juntas. Conseguir un correcto encaje de las piezas que forman la bóveda exige imperativamente el colocarlas separadas unas de otras, de esta forma, se consigue evitar que los pequeños errores de talla se vayan trasladando y multiplicando de unas a otras (Figura 18). Mediante galces interpuestos las dovelas quedan separadas unas de otras, posteriormente la junta se rellena con mortero líquido. En las construcciones antiguas, las juntas entre dovelas se suelen manifestar con toda claridad.

Una vez que la nervadura de la bóveda ha sido colocada, podría procederse al relleno de los cascos de plementería. Por regla general, la plementería de las bóveda de crucería españolas se suelen aparejar a la francesa, es decir, colocando la mampostería en arista o, dicho de otro modo, en lechos paralelos a los eje x e y. En este caso, al ser una bóveda esférica podría hacerse en vuelta de horno, es decir, por lechos horizontales, como el propio Vandelvira aconseja (Figura 20). En este caso, se ha tomado la decisión de no ejecutar la plementería con objeto de que la red de nervaduras se perciba con la mayor claridad. Una vez que todas las piezas han encontrado acomodo, se pone de manifiesto la belleza del diseño del arquitecto (Figura 22).

\section{LA ESTABILIDAD DE LA BÓVEDA}

Queda por último proceder al descimbrado de la bóveda. Como se acaba de mencionar en el párrafo anterior, se ha desestimado llevar a cabo el relleno de las plementerías, por tanto, al descimbrar, la estabilidad de la bóveda quedaría confiada únicamente a la resistencia de los arcos. La contribución de los arcos a la estabilidad de las bóvedas góticas ha sido un tema muy controvertido. El debate del papel estructural de los nervios tiene una larga trayectoria que, ni siquiera la Segunda Guerra Mundial terminaría por despejar del todo (5); el pensamiento racionalista gótico (6) en el que el nervio era parte estructural e imprescindible en la estabilidad de la bóveda, quedó en evidencia al contemplar los enormes destrozos en las catedrales francesas, con sus bóvedas en pie a pesar de haber perdido sus nervaduras; claramente se pudo observar cómo la cáscara de la bóveda era capaz de absorber incluso los esfuerzos ocasionados por los grandes agujeros de sus plementerías.

Descimbrar la bóveda de Vandelvira sin las plementerías nos permite recordar este ya antiguo debate; se trata de una ocasión irrepetible para comprobar si, solamente con la nervadura, una bóveda de este tipo podría ser estable. Durante su ejecución se había llevado a cabo un estudio de estática gráfica calculando el polígono de presiones de 


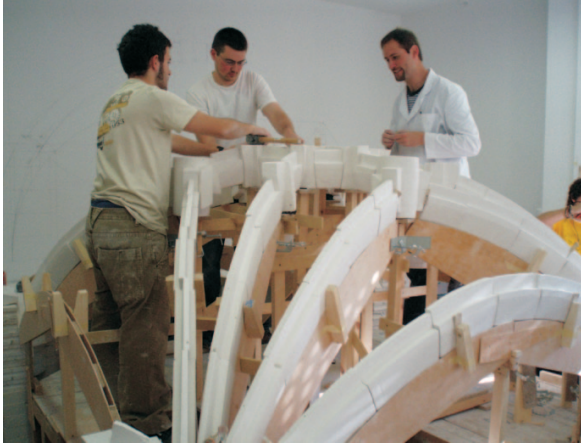

18

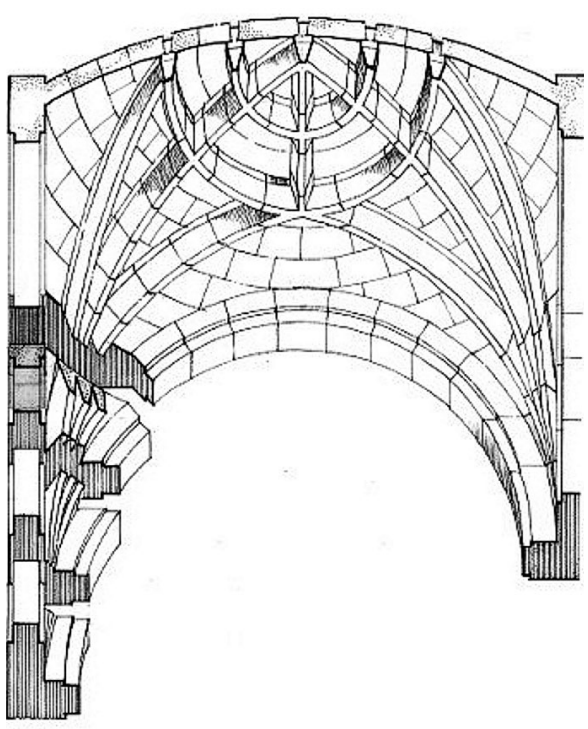

riv 1 in

cada arco (Figura 21). Este estudio permitió comprobar que, pese a la no muy favorable forma esférica de la bóveda, la línea de empujes consigue alojarse en el interior la sección de los arcos. En su recorrido, esta línea produce una tangencia en la cara de intradós del arco, lo cual anuncia riesgos de tracciones por la cara superior y, en consecuencia, la apertura de los arcos por el trasdós. Por último, vemos cómo la resultante se sale de la sección del arco, con un ángulo de $45^{\circ}$, a una altura algo inferior a la mitad de la alzada de la bóveda. El considerable peso de las jarjas seguramente será capaz de neutralizar el empuje horizontal que inevitablemente va a producirse, sin embargo, la prudencia aconseja zunchar la bóveda a esta altura. Así pues, los cálculos previos permitían aventurar que la bóveda debía ser estable, el colapso de la misma únicamente podría venir ocasionado por un defecto en su construcción². Para el descimbrado, se procedió en primer lugar a retirar los camones de los arcos ojivos, posteriormente los de los terceletes; los formeros serían descimbrados al final ya que, al faltar la plementería, quedan completamente aislados y no colaboran en la estabilidad del conjunto.

Al quedar liberados de sus cimbras, los arcos

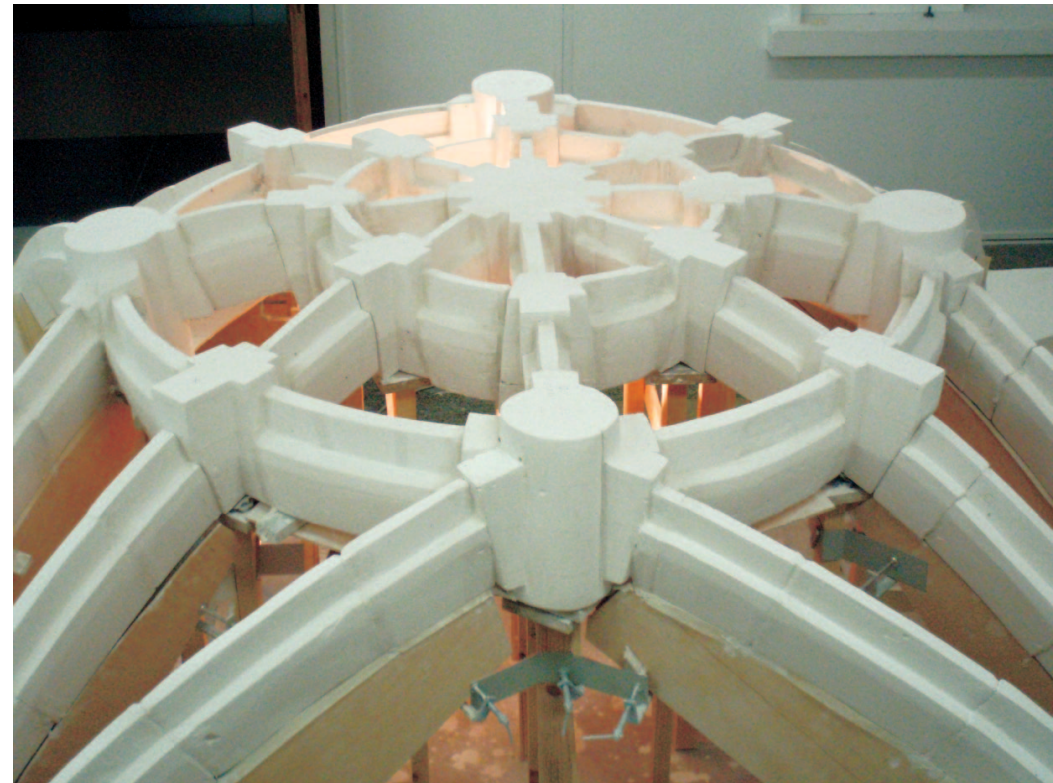

19

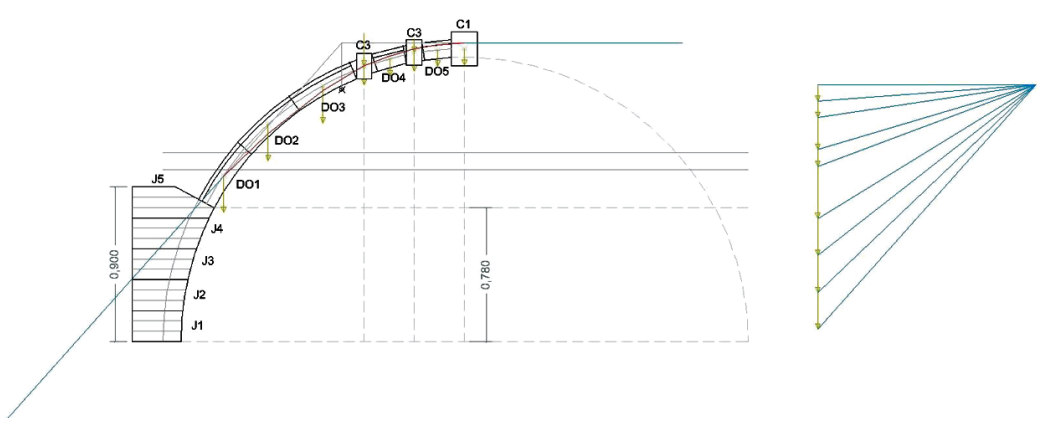

se sujetan a sí mismos, son arcos rampantes que se inician en las claves y descansan en sus apoyos. El verdadero trabajo de la bóveda todavía no ha comenzado, ese momento se producirá al quitar las cuatro cuñas que sujetan la plataforma sobre la que descansan las diecisiete claves, es entonces cuando todas ellas descenderán al mismo tiempo unos milímetros y las cargas, hasta ese momento verticales, buscaran un recorrido a lo largo de los arcos. En ese preciso momento todo el conjunto comenzara a trabajar como una bóveda.

Tradicionalmente, el descimbrado de las grandes obras de la Antigüedad era objeto de actos sociales de gran relevancia. Los prelados o incluso el rey podía desplazarse al lugar para contemplar ese acontecimiento emocionante en el que las piedras, incomprensiblemente, quedarían suspendidas en el espacio, permanecerían en el aire gracias al sabio aparejo de las mismas por el artificio de la construcción. Como entonces, también en esta ocasión, el descimbrado de la bóveda generó fuertes expectativas ${ }^{3}$. En primer lugar, se procedió a retirar las cuñas de la plataforma central, la bóveda entra entonces en carga y adquiere una casi imperceptible
18. En el montaje de la bóveda, las juntas entre las piezas requieren una especial atención.

19. El dibujo central de la bóveda, con sus dos ruedas de combados, es posible gracias a su forma esférica.

20. Al ser una bóveda de crucería esférica el aparejo de su plementería puede disponerse en vuelta de horno.

21. La estática gráfica muestra cómo la línea de empujes consigue alojarse en la sección del arco al llegar éste a la jarja.

2 La bóveda fue destruida mediante un ensayo a rotura aplicando una carga vertical en su clave central. Los resultados de este experimento desbordan los límites de este artículo por lo que serán recogidos en un artículo próximo, sin embargo, su filmación puede consultarse en www. youtube.com/upm. 
22. Una vez descimbrada la bóveda es posible apreciar la esbeltez de sus nervios y la belleza de su diseño.

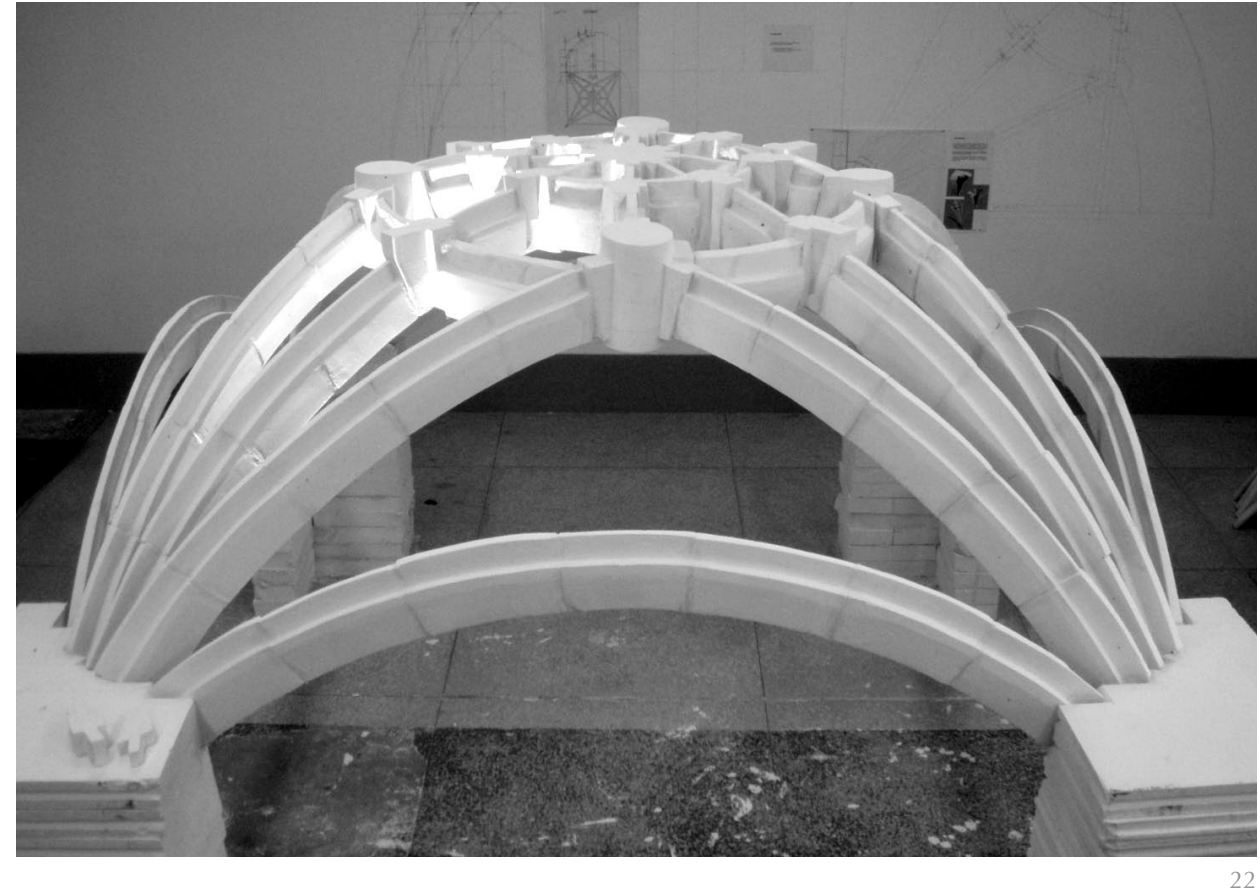

22

deformación, solamente al retirar los calzos de madera dispuestos a continuación, la cimbra se separa visiblemente de la bóveda y ésta queda claramente suspendida en el aire.

La bóveda es, por tanto, estable únicamente con la crucería.

Una vez limpia por completo, la nervadura de la bóveda se nos antoja de una extraordinaria esbeltez y pone en evidencia la belleza del diseño de Vandelvira (Figura 22). Apreciamos ahora la contundencia de su forma esférica reforzada por el dibujo de las dos ruedas concéntricas de combados, como el polo de un globo terráqueo. Una imagen que nos sumerge por completo en el espíritu del renacimiento.

\section{AGRADECIMIENTO}

Este artículo debe terminar con un especial agradecimiento a los alumnos del Taller de Construcción Gótica del curso 2008-09. Sin su entusiasmo y dedicación la bóveda jamás hubiera podido ser construida.

\section{BIBLIOGRAFÍA}

(1) Vandelvira, Alonso. “'Libro de las traças y cortes de piedra."' 1575-1580. Copias: Biblioteca Nacional de Madrid, Escuela de Arquitectura de Madrid, facsímil: Geneviève Barbé-Coquelin de Lisle: “Tratado de Arquitectura de Alonso de Vandelvira." Albacete. 1977. Caja de Ahorros.

(2) Palacios Gonzalo, José Carlos. “Trazas y Cortes de Cantería en el Renacimiento Español." Editorial Munilla-Lería. Madrid. 2003.

(3) Rabasa Díaz, Enrique. "Construcción de una bóveda de crucería en el centro de oficios de León." Actas del cuarto Congreso de Historia de la Construcción, Cádiz, vol. II. Instituto Juan de Herrera. Madrid. 2005. Del mismo autor: “Guía práctica de la estereotomía de la piedra." Centro de los Oficios de León. León. 2007.

(4) Simón García. “Compendio de arquitectura y simetría de los templos..." Colegio Oficial de Arquitectos de Valladolid, estudios introductorios de Antonio Bonet Correa y Carlos Chanfón Olmos. Valladolid. 1991.

(5) Torres Balbás, Leopoldo. “Función de nervios y ojivas en las bóvedas góticas." Investigación y Progreso, pags: 214 a 231. Madrid. 1945.

(6) Viollet-le-Duc Emmanuel. “Dictionnaire raisonné de l'architecture française du XI au XVI siècle”, 10 vols. 1854-1868. Véase del mismo autor: “'La construcción medieval." Madrid. 1996. Inst. Juan de Herrera, ETS de Arquitectura de Madrid.

(7) Caballero Zoreda, L., Latorre González-Moro, P. 1995: “Leer el documento construido”. Número monográfico de Informes de la Construcción (435).

(8) Huerta, S. 2005: “Mecánica de las bóvedas de fábrica: el enfoque del equilibrio”. Informes de la Construcción, vol. 56, nº 496 . 